\title{
Erratum to "Algorithms for U-Model-Based Dynamic Inversion (UM-Dynamic Inversion) for Continuous Time Control Systems"
}

\author{
Ruobing Li $\mathbb{D},{ }^{1}$ Quanmin Zhu $\mathbb{D}^{1},{ }^{1}$ Janice Kiely, ${ }^{1}$ and Weicun Zhang $\mathbb{D}^{2}$ \\ ${ }^{1}$ Department of Engineering Design and Mathematics, University of the West of England, Frenchy Campus, Coldharbour Lane, \\ Bristol BS16 1QY, UK \\ ${ }^{2}$ School of Automation and Electrical Engineering, University of Science and Technology Beijing, Beijing 100083, China \\ Correspondence should be addressed to Ruobing Li; 195938486@qq.com
}

Received 21 September 2020; Accepted 21 September 2020; Published 5 October 2020

Copyright (c) 2020 Ruobing Li et al. This is an open access article distributed under the Creative Commons Attribution License, which permits unrestricted use, distribution, and reproduction in any medium, provided the original work is properly cited.

In the article titled "Algorithms for U-Model-Based Dynamic Inversion (UM-Dynamic Inversion) for Continuous Time Control Systems" [1], there was an error in equation (29). Also, the Acknowledgments section was omitted in error. The corrected equation is given as follows:

$$
\left\{\begin{array}{l}
\lambda_{0}=\left(1-y^{2}\right) \dot{y}-u, \\
\lambda_{1}=1+y, \\
\lambda_{2}=1+\dot{y}^{2}, \\
\lambda_{3}=1 .
\end{array}\right.
$$

The error was introduced during the production process of the article, and Hindawi apologies for causing this error in the article. The Acknowledgments section is given as follows:

\section{Acknowledgments}

The authors would like to show their gratitude to the editors and the anonymous reviewers for their helpful comments and constructive suggestions with regards to the revision of the paper.

\section{References}

[1] R. Li, Q. Zhu, J. Kiely, and W. Zhang, "Algorithms for U-model-based dynamic inversion (UM-Dynamic inversion) for continuous time control systems," Complexity, vol. 2020, Article ID 3640210, 14 pages, 2020. 